\title{
An analytical model of information spreading through conjugation in bacterial nanonetworks
}

\author{
G. Castorina L. Galluccio S. Palazzo \\ CNIT Research Unit at Dipartimento di Ingegneria Elettrica Elettronica e Informatica, \\ University of Catania, Italy \\ email: name.surname@cnit.it
}

\begin{abstract}
Molecular communications are a powerful tool to implement communication functionalities in environments where the use of electromagnetic waves becomes critical, e.g. in the human body. Molecules such as proteins, DNA, RNA sequences are used to carry information. To this aim a novel approach relies on the use of genetically modified bacteria to transport enhanced DNA strands, called plasmids, where information can be encoded and then transferred among bacterial cell using the so called conjugation process. Information transfer is thus based on bacteria motility, i.e. self-propelled motion which under appropriate circumstances is exhibited by certain bacteria, which is still not completely understood. In this paper we propose an analytical model to characterize information spreading in bacterial nanonetworks by employing an epidemic approach similar to the one used to model Delay Tolerant Networks (DTNs) and we show that such modeling can be profitably used to represent information spreading in conjugation-based bacterial nanonetworks
\end{abstract}

\section{Keywords}

Information spreading, bacterial nano networks.

\section{INTRODUCTION}

In molecular communications information is encoded into molecules (e.g. proteins, DNA and RNA strands, pheromones, etc.) and, specifically, in their type, number, concentration or release time. In turn, molecular communications can be classified depending on the distance between sender and receiver [4] or, according to the transport mechanism used by the molecules. In the latter case we distinguish between active and passive transport techniques. Passive transport techniques do not need for external energy to move molecules and rely on diffusive phenomena (e.g. Brownian motion, diffusion theory). Active transport techniques need for external energy supply and imply the use of molecular motors, catalytic nanomotors and flagellated bacteria (suitable to transport genetic information due to the high motility given

P ermission to make digital or hard copies of all or part of this work for personal or classroom use is granted without fee provided that copies are not made or distributed for profit or commercial advantage and that copies bear this notice and the full citation on the first page. To copy otherwise, to republish, to post on servers or to redistribute to lists, requires prior specific permission and/or a fee.

BODYNETS 2015, September 28-30, Sydney, Australia

Copyright $\odot$ 2015 ICST 978-1-63190-084-6

DOI 10.4108/eai.14-12-2015.151107 by their flagella). Several examples exist in the literature that describe architectures for active transport molecular communications based on the use of molecular motors and flagellated bacteria. Nevertheless none of these works provides a mathematical model to study information propagation in nanonetworks based on active transport techniques and, in particular, flagellated bacteria. An analytical model for delay and capacity in bacteria-based nanonetworks is derived in [2], in which bacteria are supposed to be relayed directly from nanomachine to nanomachine, but no mention to opportunistic conjugation, i.e. transfer of plasmids between bacterial cells, is available. Furthermore, in the analytical model, delay is split into a fixed delay and a stochastic propagation delay that is studied through simulations. In this paper we introduce an architecture derived from the one proposed in [4] and [5] and use an epidemic model to analytically characterize bacteria diffusion and the plasmids dissemination in molecular networks. In particular, we evaluate time delivery and loss performance in these scenarios by using epidemic approaches inherited by DTN's modeling. The rest of this paper is organized as follows. In Section 2 we describe the related work and give the necessary background to understand the proposed model. In Section 3 we describe the architecture of the proposed model and derive a mathematical description. In Section 4 numerical results are provided and discussed. In Section 5 conclusions are drawn.

\section{RELATED WORK}

Bacteria are naturally able to spread genetic information across a colony through a process known as conjugation. The conjugation process takes place between pairs of bacteria that come into each other proximity and allows to exchange the genetic information contained in specific double stranded circular DNA sequences, known as plasmids, which are generally free to move inside the bacterial cytoplasm. A plasmid, normally, does not include functionalities necessary for the survival of the bacterium, but gives it some extra-ability (e.g. antibiotic resistance, virulence or metabolic property) useful to proliferate in particular conditions. Plasmids can be classified into incompatibility groups so that different plasmids can coexist inside the cellular body as long as they are not in the same incompatibility group. When a bacterium with a specific plasmid comes in proximity of another one both having no plasmids of the same incompatibility group, the conjugation process starts. As an example consider the F-plasmid (Fertility factor). The F-plasmid is responsible for the formation of a sex pilus used to initiate the conjugation process. When a bacterium pro- 


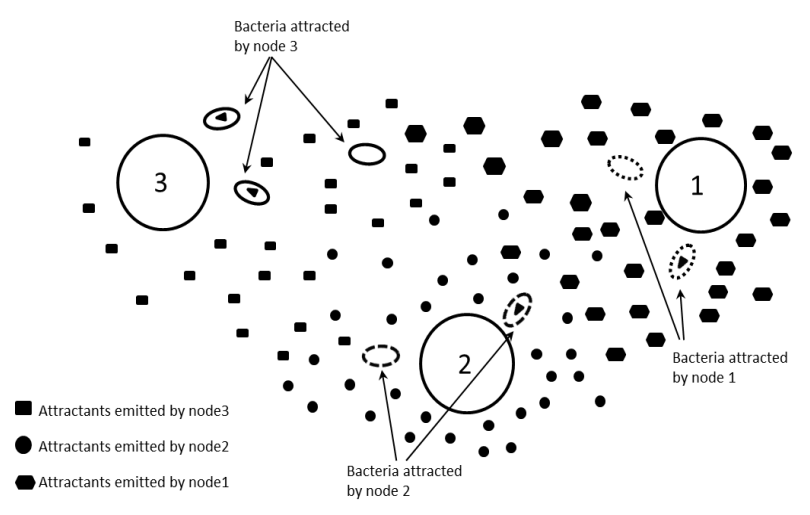

Figure 1: Example of bacterial nanonetwork

vided with the $\mathrm{F}$-plasmid $\left(\mathrm{F}^{+}\right.$bacterium) is close enough to a bacterium without the F-plasmid ( $\mathrm{F}^{-}$bacterium), a sex pilus extends from the donor $\left(\mathrm{F}^{+}\right.$bacterium $)$to the recipient ( $\mathrm{F}^{-}$bacterium). Thus, due to the sex pilus contraction the two cellular membranes get in contact and a channel is opened to allow the passage of the F-plasmid from the $\mathrm{F}^{+}$ to the $\mathrm{F}^{-}$bacterium.

In [4] a flagellated bacteria-based paradigm was proposed to implement medium range molecular communications. Bioengineering techniques can be used to create artificial mutated bacteria with chimeric plasmids (i.e. in vitro built up plasmids) in order to form a dictionary of bacteria with a set of possible messages encoded in chimeric plasmids.

Motility of flagellated bacteria relies on helical movements of the flagella. Through several pili distributed along the cellular body, bacteria can sense concentration of nutrients in the environment and decide to move towards points with an increasing concentration level. This process is known as chemotaxis.

Concerning the network model in [4], it consists of several nanomachines which emit chemoattractants to attract bacteria from the environment (Figure 1). Different nodes (i.e. nanomachines) can spread different types of attractants so that they can stimulate a different bacterium. Furthermore, any node can store a set of bacteria that can carry an information packet in the form of a plasmid and can be attracted by neighbors nodes. A node that wants to send a packet to another nanomachine will release bacteria with the encoded packet into the environment. Through the chemotaxis process, bacteria will find their own way to neighbor nodes emitting the appropriate chemical compound and will deliver the packet.

An opportunistic routing scheme based on bacterial conjugation was proposed in [5]. Bacteria used to transport the information are supposed to be E. Coli and it is assumed that node-to-node paths are followed by bacteria to cross each other. If a bacterium, with a packet encoded into a plasmid, meets another one that is not carrying any message (no plasmid), conjugation will be executed so that at the end of the process both bacteria will transport the same packet. As an example consider the system shown in Figure 2: where a bacterium, sent from the nanomachine 1 (node 1 ) to node 2 , in proximity of node 2 will eventually meet bacteria directed from node 2 to node 3 ; thus, by exploiting the conjugation process, the message will be relayed to node
3.

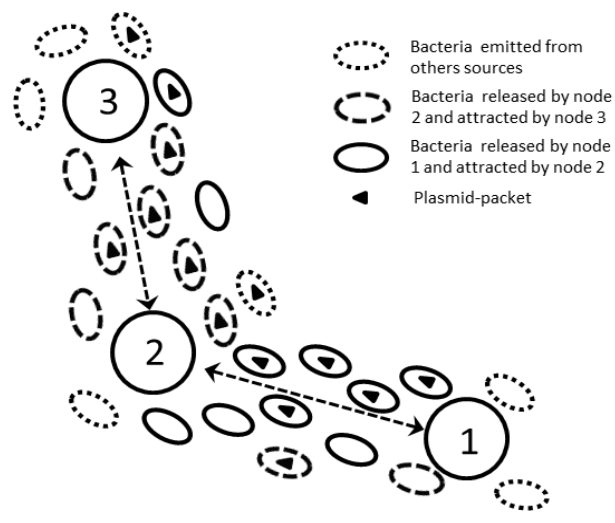

Figure 2: Opportunistic routing through conjugation

\section{SYSTEM MODEL}

We consider bacteria as mobile nodes that occasionally come into each other proximity and exchange information. Such a model is similar to those used for epidemic spreading in Delay Tolerant Networks (DTNs) [3], [8], [7]. In DTNs, an infection disease model, known as SIR [6], is usually exploited to describe the spreading of information among a population of mobile nodes. In the SIR models the total population is assumed constant [6] and three different classes of individuals are considered: individuals susceptible to be infected, $S$, infected individuals, $I$ and recovered individuals, $R$ (individuals that have been infected and then recovered through a vaccine or immunization process). In our model we initially consider that only one packet (i.e. plasmid) is flowing into the network issued by a source nanomachine. Each bacterium represents a mobile node (node-M). A bacterium that has no plasmid (i.e. packet) is considered susceptible (node-S) to be infected; a bacterium with a plasmid (a packet) is considered infected (node-I). Moreover, a destination node (node-D) is a nanomachine able to connect to a bacterium through a conjugation process, receive the plasmid and de-activate the bacterium that is then considered recovered (node-R). Relay nanomachines are available and can issue bacteria.

Bacteria released by the source will move randomly and eventually meet other bacteria, start conjugation and make the packet spread into the area. This process continues until one or more bacteria with the encoded plasmid reach the destination node. The destination nanomachine will be able to receive the packet and kill the bacterium. We consider a mathematical model that assigns destinations as static nodes, bacteria in the environment as mobile nodes and a static source at time $t=0$.

\subsection{Plasmid Spreading Model}

We consider the case when a single plasmid can be carried by a node-M. We refer to the F-plasmid transfer model as an example and every nanomachine emits attractants, sensed by bacteria of any node-M. Attractants concentration can be approximated as uniform into the network area. Let $\mathrm{I}(\mathrm{t})$ be the number of infected nodes at time $t, S(t)$ the number of susceptible nodes at time $t$ and $R(t)$ the number of 


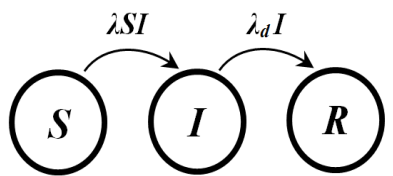

Figure 3: Markov chain of a SIR infection model

recovered nodes at time t. We assume that inter-contact time between bacteria is exponentially distributed. Let $\lambda$ be the inter-contact rate between a pair of bacteria and let $\lambda_{d}$ be the inter-contact rate between bacteria and destination nodes. Under these assumptions, the model can be described as a Markov chain where a transition occurs every time an infected node meets a non infected node (i.e. I(t) increases) or recovery occurs after an infected node reaches a node-D (I(t) decreases). If $N$ is the average number of nodes (bacteria) and $D$ the number of destinations, the plasmid diffusion process can be modeled as:

$$
I^{\prime}(t)=[N-I(t)-R(t)] \lambda I(t)-R^{\prime}(t)
$$

and

$$
R^{\prime}(t)=D \lambda_{d} I(t)
$$

For worth of illustration we consider a static topology of nanomachines like the one plot in Figure 4: nanomachine 8 is the source node, the nano-machine 3 is the destination node. When a node needs to send a packet it will release a certain amount of bacteria (i.e. a given rate) with the same encoded message (plasmid). Due to the uniform attractants concentration, bacteria move randomly in the area with no preferential path. So they can be modeled as $N$ nodes-M that meet each other and the destination nodes with intercontact rates $\lambda$ and $\lambda_{d}$, respectively. To derive the CDF of the first delivery time, we can observe that no recovery can occur until the first packet is delivered to a destination. Under this assumption eq. (1) assumes the following form:

$$
I^{\prime}(t)=[N-I(t)] \lambda I(t)
$$

and eq. (3) is an ODE equation with initial condition $I(0)=$ 1 that has the following solution:

$$
I(t)=\frac{N}{1+e^{-\lambda N t}(N-1)}
$$

Furthermore, if $T$ is the time from the generation of the packet until the first time destination receives it, by considering a single destination, the cumulative probability distribution for $T, F_{T}(t)$, can be computed as in [7]:

$$
F_{T}(t)=1-K\left(\frac{N-1}{e^{\lambda N T}+N-1}\right)^{\frac{\lambda_{d}}{\lambda}}
$$

with $K=\left[\frac{N-1}{N}\right]^{-\frac{\lambda_{d}}{\lambda}}\left[1-F_{T}(0)\right]$

\section{NUMERICAL ANALYSIS}

In this section, we illustrate the numerical results obtained by applying the proposed analytical model. We analyze the plasmid spreading model given the number of bacteria in the environment, the inter-contact rates among bacteria and the inter-contact rates between bacteria and destinations. To simplify the analysis we consider a network topology like

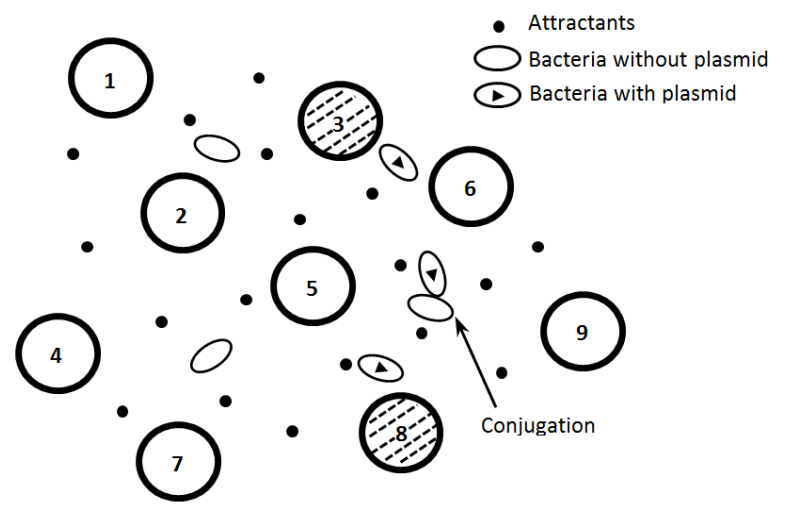

Figure 4: Static topology with a single attractants uniformly distributed at random

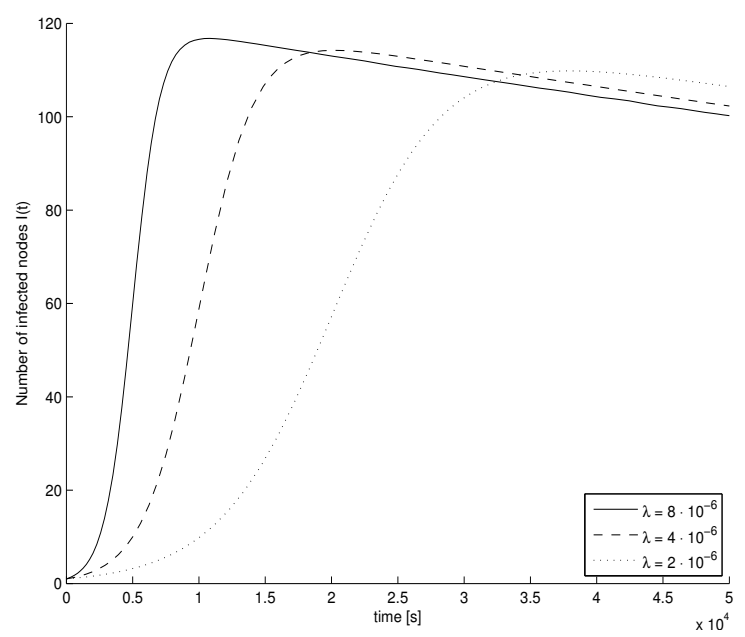

Figure 5: Number of infected nodes vs time for a single plasmid dissemination with a single attractant

the one plotted in Figure 2. For the plasmid model we set $N=120$ bacteria, i.e. nodes-M, that are supposed to be uniformly distributed at random. Nanomachine 1 is assigned as the source nanomachine, nanomachine 3 as the destination nanomachine and nanomachine 2 as the relay nanomachine. Furthermore one infected bacterium is set as initial condition. The plasmid spreading model is analyzed for different values of $\lambda$ and $\lambda_{d}$. The inter-contact rate $\lambda$ is obtained from data reported in [1] and is set as $\lambda=8 \cdot 10^{-6}$. Figure 5 shows the number of infected nodes-M (bacteria) as a function of time $t$. The CDF of $F_{T}(t)$ is computed for different values of $\lambda_{d}$ and is plotted in Figure 6. By increasing the intercontact rate between bacteria and destinations the delivery time decreases. In Figure 7 we report the average first delivery time versus the ratio between $\lambda$ and $\lambda_{d}$. As expected the number of infected nodes (Figure 5) starts to increase in a non linear way and reaches a maximum value; then the recovery process becomes dominant and the number of infected nodes decreases. Note that, the maximum number of infected nodes (Figure 5) is very close to the total number of bacteria distributed in the environment; for this reason, as can be observed also in Figure 7, the recovery process immediately starts to prevail after the first delivery. This behavior 


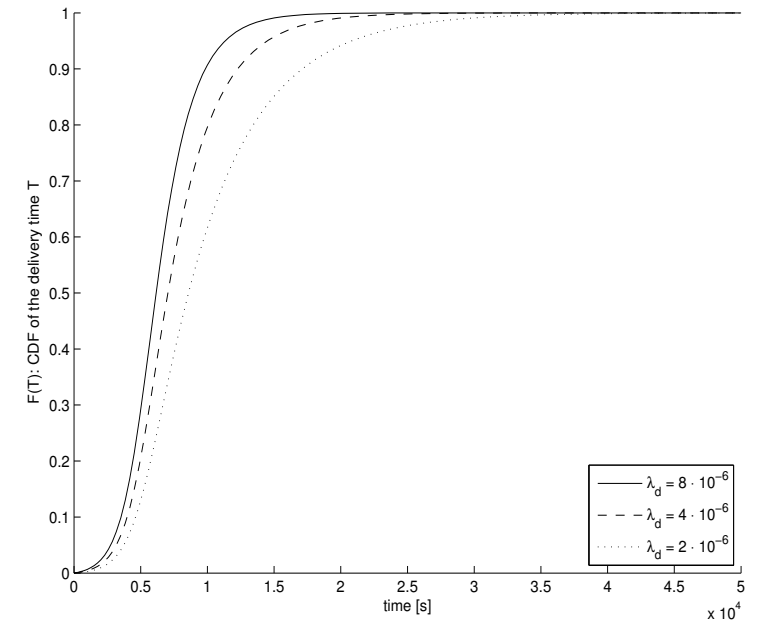

Figure 6: CDF of the delivery time $T$ for a single plasmid dissemination with a single attractant

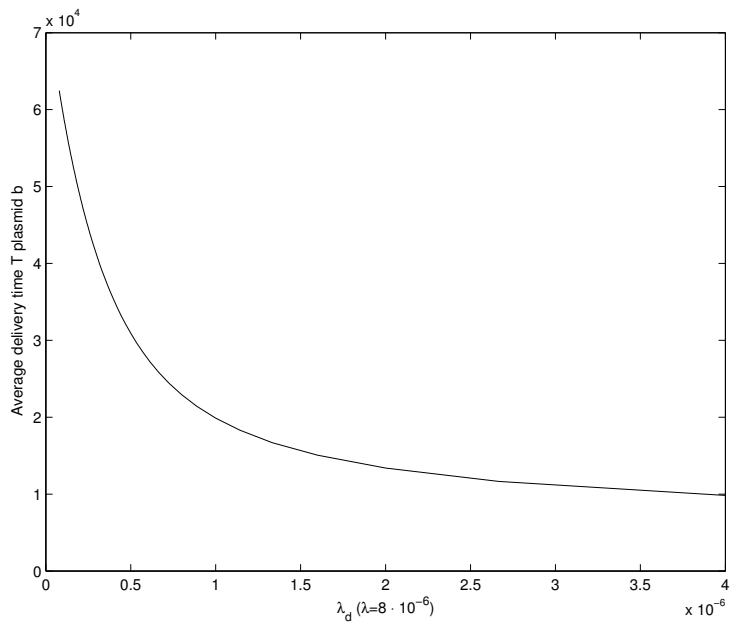

Figure 7: Average delivery time $\mathrm{T}$ for a single plasmid dissemination with a single attractant

suggests that the probability to deliver a packet is very low even for high number of infected nodes, but rapidly increases upon approaching the saturation of the network (number of infected nodes equal to the total number of bacteria in the environment). Finally, in Figure 8, we show the number of recovered nodes versus time. As expected the number of recovered nodes increases in a non linear way and we observe that the proposed recovery scheme is a very slow process if compared to the diffusion of the infection.

\section{CONCLUSIONS}

Bacteria-based communications have been recently proposed as a promising approach to implement middle range communications and support networking functionalities in molecular scenarios. In this paper we presented an analytical model to characterize the behavior of multi-hop communications in bacterial nanonetworks that take into account conjugation processes. In particular we presented a model to describe a pure epidemic routing; we set a simple test case consider-

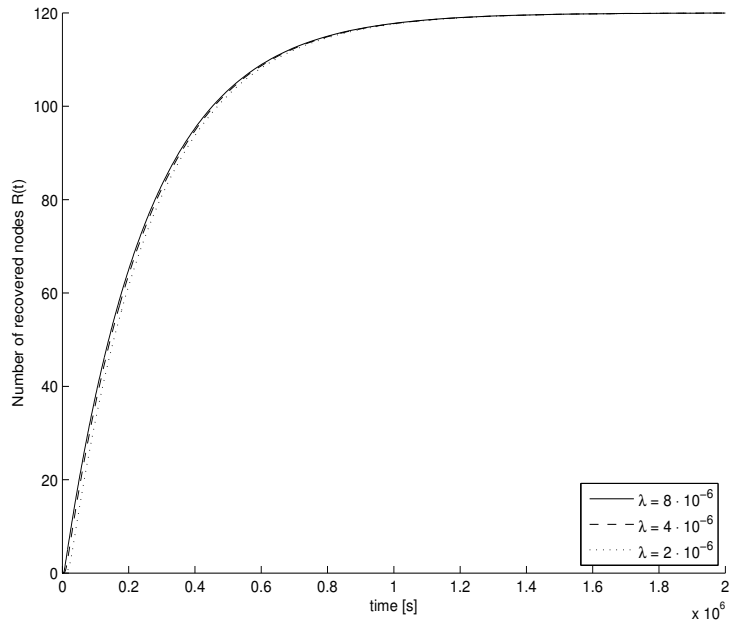

Figure 8: Number of recovered nodes vs time for a single plasmid dissemination with a single attractant

ing a topology with 3 nodes and we derived the numerical solution. The model showed a strong dependence on the inter-contact rate among bacteria and between bacteria and destination. We think that the epidemic approach represents a simple, feasible and reliable solution for modeling of information vive spreading in conjugation-based bacterial nanonetworks.

\section{REFERENCES}

[1] S. Balasubramaniam and P. Lio. Multi-hop conjugation based bacteria nanonetworks. IEEE Trans. on Nanobioscience, 12(1):47-59, March 2013.

[2] L. C. Cobo and I. F. Akyildiz. Bacteria-based communication in nanonetworks. Nano Commun. Netw., 1(4):244-256, December 2010.

[3] L. Galluccio, B. Lorenzo, and S. Glisic. Sociality-aided new adaptive infection recovery schemes for multicast dtns. Accepted for publication in a future issue of IEEE Transactions on Vehicular Technology, 2015.

[4] M. Gregori and I. F. Akyildiz. A new nanonetwork architecture using flagellated bacteria and catalytic nanomotors. IEEE Journal on Sel. Areas in Comm., 28(4):612-619, May 2010.

[5] P. Lio and S. Balasubramaniam. Opportunistic routing through conjugation in bacteria communication nanonetwork. Nano Commun. Netw., 3(1):36-45, March 2012.

[6] J. Murray. Mathematical Biology: I. An Introductioned. 3rd ed. Interdisciplinary applied mathematics, vol. 17. Springer, New York, 2001.

[7] T. Small and Z. Haas. The shared wireless infostation model - a new ad hoc networking paradigm. In MobiHoc. ACM, 2003.

[8] J. K. D. T. X. Zhang, G. Neglia. Performance modeling of epidemic routing. Computer Networks, 51(10):2867-2891, July 2007. 